\title{
Diffuse Pancreatic Inflammation in an HIV Infected Individual With Elevated IgG4 Levels
}

\author{
Abhishek Bhurwal $^{\mathrm{a}}$, Sunil Sapru ${ }^{\mathrm{a}}$, Dhanasekaran Ramasamy ${ }^{\mathrm{b}}$
}

\begin{abstract}
Even though, acute pancreatitis is a frequent complication of human immunodeficiency virus (HIV) infection and anti-retroviral therapy, pancreatic inflammation directly related to HIV infection is uncommon and is difficult to diagnose. The presence of features suggestive of autoimmune etiology complicates the diagnosis making process even more difficult. Rarely, HIV-related inflammation of pancreas mimicking radiological features of autoimmune pancreatitis has been described. However, serological features of autoimmune pancreatitis have not been described in HIV-related inflammation of pancreas. Here, we present a case of acute pancreatitis in an HIV patient where the etiology was attributed to HIV-related inflammation after extensive workup despite serological features suggestive of autoimmune pancreatitis. Therefore, the case report suggests that the physician should consider other etiologies prior to labelling autoimmune pancreatitis based on serology alone.
\end{abstract}

Keywords: Acute pancreatitis; HIV; IgG4

\section{Introduction}

Acute pancreatitis is a well-recognized complication of human immunodeficiency virus (HIV) infection as well as antiretroviral therapy. HIV-related inflammation of the pancreas is an infrequent cause of acute pancreatitis [1-4]. The presence of confounding factors such as features suggestive of autoimmune disease makes the diagnosis difficult. However, HIV-related inflammation of the pancreas sharing features with autoimmune pancreatitis has rarely been reported [5]. Serological features (elevated IgG4) reported in autoimmune pancreatitis have not been demonstrated in HIV-related in-

Manuscript submitted January 28, 2018, accepted February 8, 2018

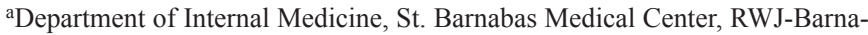
bas Health, NJ, USA

${ }^{b}$ Department of Internal Medicine, Division of Gastroenterology, St. Barnabas Medical Center, RWJ-Barnabas Health, NJ, USA

${ }^{\mathrm{c} C}$ Corresponding Author: Dhanasekaran Ramasamy, Center for Digestive Diseases, 695 Chestnut Street, Union, NJ 07083, USA.

Email: anudhana@hotmail.com

doi: https://doi.org/10.14740/jmc3012w flammation of pancreas. It is important for the physician to carefully consider the diagnosis of autoimmune pancreatitis in immunocompromised patients as the use of steroids may lead to adverse events. Here, we present a case of acute pancreatitis in an HIV patient where the etiology was attributed to HIV-related inflammation after extensive workup despite serological features described in autoimmune pancreatitis. The case report emphasizes the importance of extremely cautious consideration of the diagnosis of autoimmune pancreatitis solely on presence of elevated IgG4 levels especially in patients with HIV infection.

\section{Case Report}

A 36-year-old HIV positive African American female presented at our emergency department for recurrent episodes of epigastric pain for the last 8 days. The pain was intermittent in nature and mild to moderate in intensity. Even though, the patient reported nausea associated with abdominal pain, she denied any episodes of vomiting. She denied any history of fever, night sweats during the last few months. She had lost 5 $\mathrm{lb}$ during the last 6 months. She denied alcohol intake prior to the episode. The patient had a history of poor medication compliance to highly active antiretroviral therapy (HAART). Due to high viral load and poor medication compliance, she had recurrent episodes of cutaneous herpetic lesions. She was also treated for candida esophagitis. She was not on any medications prior to the visit. Physical examination was unremarkable except for epigastric tenderness. Initial investigations revealed lipase of 1,367 U/L (normal 3 - $60 \mathrm{U} / \mathrm{L}$ ). Liver function tests, calcium levels and triglyceride levels were within normal limits. The plasma HIV viral load was $1,383,810$ copies RNA/ $\mathrm{mL}$ and CD4 count was 20 cells $/ \mathrm{mm}^{3}$. Contrast enhanced computed tomography (CT) scan of the abdomen showed diffuse inflammatory changes consistent with acute pancreatitis (Fig. 1) but further imaging did not reveal capsule-like rim or lymph node enlargement (Fig. 2). The patient's complaints, elevated levels of lipase along with imaging features were consistent with acute pancreatitis. Subsequently, treatment including IV fluids, pain management and bowel rest was initiated. However, the etiology of the pancreatitis was unable to be determined initially. The usual causes such as alcohol intake, medications, and gallbladder stones were not present in our patient. The low CD4 count prompted workup for possible opportunistic infections of the pancreatitis. Surprisingly, evaluation for infectious etiologies was unrevealing, including syphilis (RPR negative), 


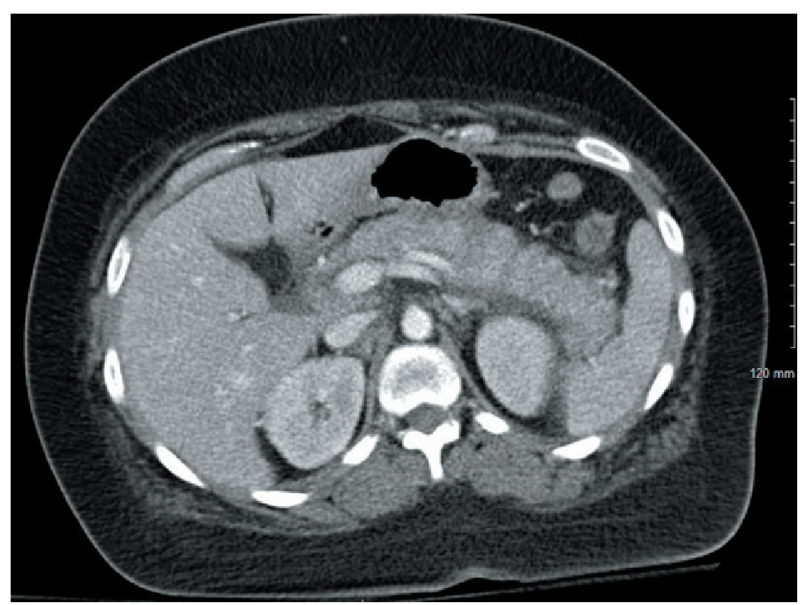

Figure 1. CT abdomen with contrast showing diffusely enlarged pancreas and peripancreatic inflammatory changes.

CMV (CMV quantitative PCR undetectable), Tuberculosis (AFB blood cultures negative), Mycoplasma (IgM negative), Histoplasma (antigen negative), and Cryptococcus (antigen negative). Further workup to determine the etiology revealed elevated IgG4 levels (117 mg/dL, normal $4-86 \mathrm{mg} / \mathrm{dL})$. The patient refused to undergo EUS for tissue diagnosis. The differential diagnosis after the workup included HIV-related inflammation, autoimmune pancreatitis. An EUS-guided biopsy could not be performed as patient refused due to personal reasons. She was treated initially with a trial of steroids for possible autoimmune pancreatitis. However, the patient's condition worsened as she continued to have abdominal pain and steroids were discontinued. Thereafter, HAART therapy was started considering the diagnosis of HIV-related inflammation of the pancreas. Subsequently, the patient started to improve and 2 months after her initial presentation, the patient was totally asymptomatic.

\section{Discussion}

This case report describes a patient who developed inflammatory changes in the pancreas which were most likely a consequence of HIV infection after undergoing an extensive workup. The incidence of pancreatitis in HIV patients is higher than general population [6] and risk increases with progression of the HIV [7]. The etiologies of acute pancreatitis in HIV-infected patients, in addition to common causes, include the side effects of the medication and opportunistic infections [8]. Inflammation of the pancreas directly related to HIV has been suggested in a few reports [1-4]. The mechanism of acute pancreatitis in such cases could be related to inflammatory process related to viral replication. Abdominal magnetic resonance imaging (MRI) or CT in HIV-infected patients has revealed non-specific features of acute or chronic pancreatitis [9]. Serum IgG4 in HIV patients has been reported to be low as compared to general population [10]. Even though, serum IgG4 was elevated, autoimmune pancreatitis was unlikely the etiology in our patient as the patient did not show other

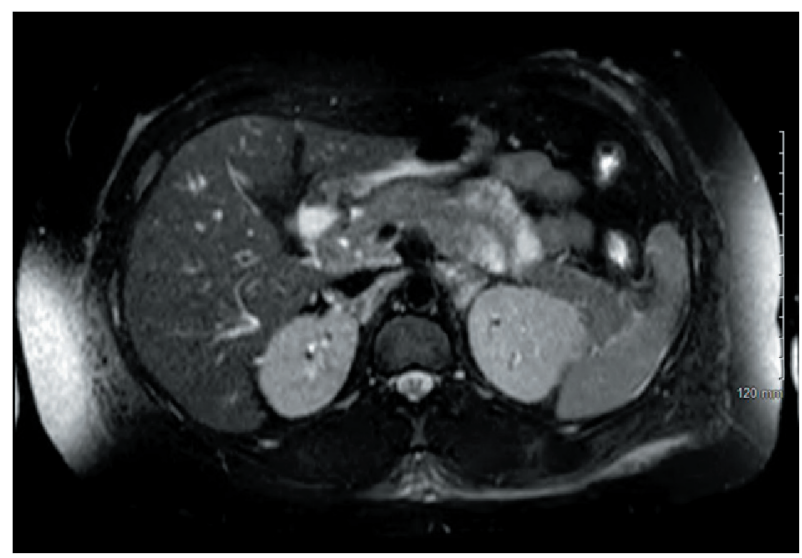

Figure 2. MRI abdomen with contrast showing no capsular rim.

features of IgG4-related disease such as salivary or lacrimal gland enlargement and lymphadenopathy. The absence of tissue diagnosis and genetic analysis such as SPINK or PRSS1 to support the etiology is a major limitation in our case report. However, we are cautious in reporting that our case most likely represents pancreatitis as a direct consequence of HIV infection after the exclusion of any opportunistic infection, side effects of the medication or malignancy. The complete resolution of the clinical abnormalities after HAART re-initiation also suggests the inflammation was related to HIV infection. To the best of our knowledge, HIV-related inflammation of pancreas mimicking radiological features of autoimmune pancreatitis has been described only once before [5]. This is only the second reported case of pancreatic inflammation which shared overlapping features of autoimmune pancreatitis in a patient with HIV and the first to report elevated IgG4 levels in these settings. However, the case suggests that the results of IgG4 should be considered in the setting of other clinical findings suggestive of autoimmune disease. Considering the diagnosis solely on basis of serological markers may lead to inappropriate treatment with steroids and increasing risks of adverse events in setting of immunocompromised patients. We also suggest that the physician should also consider HIV infection leading to inflammation of the pancreas as the etiology of pancreatitis in patients with poorly controlled HIV infection.

\section{Author Contributions}

Abhishek Bhurwal contributed towards data gathering and writing the manuscript. Sunil Sapru and Dhanasekaran Ramasamy contributed equally towards manuscript editing. D. Ramasamy also contributed towards data gathering.

\section{Financial Support}

We certify that we have no financial affiliation/interest (e.g., employment, stock holdings, consultant arrangements, and honoraria in the subject matter, materials, or products men- 
tioned in this manuscript).

\section{Declaration}

Informed consent was taken from the patient for writing the manuscript.

\section{References}

1. Rizzardi GP, Tambussi G, Lazzarin A. Acute pancreatitis during primary HIV-1 infection. N Engl J Med. 1997;336(25):1836-1837.

2. Mortier E, Gaba S, Mari I, Vinceneux P, Pouchot J. Acute pancreatitis during primary HIV-1 infection. Am J Gastroenterol. 2002;97(2):504-507.

3. Tyner R, Turett G. Primary human immunodeficiency virus infection presenting as acute pancreatitis. South Med J. 2004;97(4):393-394.

4. Pano-Pardo JR, Alcaide ML, Abbo L, Dickinson G. Primary HIV infection with multisystemic presentation. Int J Infect Dis. 2009;13(4):e177-180.

5. Leurquin-Sterk G, Schepers K, Delhaye M, Goldman
S, Verset L, Matos C. Diffuse pancreatic lesion mimicking autoimmune pancreatitis in an HIV-infected patient: successful treatment by antiretroviral therapy. JOP. 2011;12(5):477-481.

6. Dutta SK, Ting CD, Lai LL. Study of prevalence, severity, and etiological factors associated with acute pancreatitis in patients infected with human immunodeficiency virus. Am J Gastroenterol. 1997;92(11):2044-2048.

7. Smith CJ, Olsen CH, Mocroft A, Viard JP, Staszewski S, Panos G, Staub T, et al. The role of antiretroviral therapy in the incidence of pancreatitis in HIV-positive individuals in the EuroSIDA study. AIDS. 2008;22(1):47-56.

8. Trindade AJ, Huysman A, Huprikar SS, Kim MK. A case study and review of pancreatitis in the AIDS population. Dig Dis Sci. 2008;53(10):2616-2620.

9. Bilgin M, Balci NC, Erdogan A, Momtahen AJ, Alkaade S, Rau WS. Hepatobiliary and pancreatic MRI and MRCP findings in patients with HIV infection. AJR Am J Roentgenol. 2008;191(1):228-232.

10. Abbas A, Vasilescu A, Do H, Hendel H, Maachi M, Goutalier FX, Regulier EG, et al. Analysis of IGG and IGG4 in HIV-1 seropositive patients and correlation with biological and genetic markers. Biomed Pharmacother. 2005;59(1-2):38-46. 